\title{
1 The genetic population structure of Lake Tanganyika's Lates 2 species flock, an endemic radiation of pelagic top predators
}

4 Jessica A. Rick ${ }^{* 1}$, Julian Junker ${ }^{\star 2,3}$, Ismael A. Kimirei ${ }^{4}$, Emmanuel A. Sweke ${ }^{4,5}$, Julieth B.

5 Mosille $^{4}$, Christian Dinkel ${ }^{2}$, Salome Mwaiko ${ }^{2,3}$, Ole Seehausen ${ }^{2,3}$, Catherine E. Wagner ${ }^{1}$

6

$7 \quad$ * denotes equal contribution

8

$9{ }^{1}$ Department of Botany and Program in Ecology, University of Wyoming, 1000 E University 10 Dr., Laramie, Wyoming 82072 USA

$11{ }^{2}$ EAWAG Swiss Federal Institute of Aquatic Science and Technology, $\mathrm{CH}-6047$

12 Kastanienbaum, Switzerland

$13{ }^{3}$ Division of Aquatic Ecology \& Evolution, Institute of Ecology \& Evolution, University of Bern, $14 \mathrm{CH}-3012$ Bern, Switzerland

$15{ }^{4}$ Tanzania Fisheries Research Institute (TAFIRI), Dar es Salaam, Tanzania

$16{ }^{5}$ Deep Sea Fishing Authority (DSFA), Zanzibar, Tanzania

17 Corresponding authors: Jessica Rick (irick@uwyo.edu) and Catherine E. Wagner

18 (Catherine.Wagner@uwyo.edu)

19

Running title: Population genetics of Lates fishes 


\section{Abstract}

23 Life history traits are important in shaping gene flow within species and can thus determine

24 whether a species exhibits genetic homogeneity or population structure across its range.

25 Understanding genetic connectivity plays a crucial role in species conservation decisions,

26 and genetic connectivity is an important component of modern fisheries management in

27 fishes exploited for human consumption. In this study, we investigated the population

28 genetics of four endemic Lates species of Lake Tanganyika (Lates stappersii, L. microlepis,

29 L. mariae and L. angustifrons), using reduced-representation genomic sequencing methods.

30 We find the four species to be strongly differentiated from one another (mean interspecific

$\left.31 \quad F_{S T}=0.665\right)$, with no evidence for contemporary admixture. We also find evidence for high

32 levels of genetic structure within L. mariae, with the majority of individuals from the most southern sampling site forming a genetic group distinct from the individuals at other sampling sites. We find evidence for much weaker structure within the other three species, $L$. stappersii, L. microlepis, and L. angustifrons, despite small and unbalanced sample sizes and imprecise geographic sampling locations, suggesting the possibility for further structure undetected in our study. We call for further research into the origins of the genetic differentiation that we observe in these four species, particularly that of $L$. mariae, which may be important for the conservation and management of this species.

Keywords: Lates angustifrons, Lates mariae, Lates microlepis, Lates stappersii, population 


\section{Introduction}

Life history traits, including reproduction, growth and dispersal, strongly influence gene flow and the genetic structure of populations (Ellegren \& Galtier 2016; Manier \& Arnold 2006; Stearns 1992). Differences in life history traits can also explain why sympatric and ecologically similar taxa may differ in their genetic population structure (Peterman et al. 2015; Young et al. 2015). Understanding how life history traits like dispersal, reproduction, and growth shape gene flow is therefore crucial to improving our understanding of local adaptation, speciation, persistence of populations, migration, and the spatial scale of effective management (Sunday et al. 2014).

In teleost fishes, traits such as spawning location, philopatry, timing of spawning, and duration of larval stage, have been shown to shape population structure (Olsen et al. 2011; Pettersson et al. 2019; Young et al. 2015). Anadromous salmonids are well-known for displaying homing behavior that leads to genetic distinction between populations that use different spawning grounds at different times, dividing populations into many genetic units despite the populations spending most of their lives together in the ocean (Wenburg et al. 1998; Brannon et al. 2004). Divergence between populations caused by temporal differences in the timing of spawning has also been observed in Atlantic herrings (Martinez Barrio et al. 2016; Pettersson et al. 2019). In the oceans, coral reef fishes which spawn on or near the substrate, which exhibit parental care strategies, and which lack planktonic larvae, generally have significantly greater population structure than pelagic spawners or substrate spawners with planktonic larvae (Riginos et al. 2014). Additionally, a shorter larval phase among pelagic spawners can lead to a more structured population, because this leads to a shorter larval dispersal distance (Selkoe \& Toonen 2011).

Understanding the genetic population structure of species is essential for conservation and sustainable fisheries management (Reiss et al. 2009; Bernatchez 2016; Supple \& Shapiro 2018). Disregarding population structure and managing a population complex as a single population can lead to overfishing or even extinction of the more vulnerable genetic groups (Reiss et al. 2009; Sterner 2007). In species with cryptic diversity, unintentional overfishing of distinct populations can lead to a loss of genetic diversity in the species as a whole. Stock diversity can contribute to the resilience of a fishery to environmental fluctuations (Schindler et al. 2015) and the loss of differentiated populations can compound to major losses in the consistency of fisheries yields (Hutchinson 2008; Schindler et al. 2010). The loss of population diversity can also have drastic effects on ecosystem services and species persistence, and understanding the extent to which population structure exists is crucial for predicting these consequences (Schindler et al. 2015; Schindler et al. 2010; Therkildsen et al. 2013). It is therefore essential to understand the extent and nature of population genetic 
structure and differential adaptation in fishes that are important food and economic resources.

\section{The pelagic fish community and the fishery of Lake Tanganyika}

The pelagic fish of Lake Tanganyika are one such fishery. Lake Tanganyika is the second largest inland fishery on the continent of Africa (Van der Knaap et al. 2014). During the last few decades, fish catches in Lake Tanganyika's fishery have exhibited a general downward trend (Kimirei et al. 2008; Van der Knaap 2013; Van der Knaap et al. 2014; van Zwieten et al. 2002). This decline has resulted from a combination of an increase in the number of fishermen and vessels on Lake Tanganyika, changes in fishing practices (e.g., the use of beach seining, which targets fish in their in-shore nursery habitats) (Kimirei et al. 2008; Petit \& Shipton 2012; Van der Knaap 2013; Van der Knaap et al. 2014; van Zwieten et al. 2002), and warming lake surface temperatures (Cohen et al. 2016; O'Reilly et al. 2003; Sayers et al. 2020). Decreases in fish abundance are likely also linked to reduced productivity in the lake caused by stronger water column stratification due to climate change (O'Reilly et al. 2003; Verburg et al. 2003). In addition, anthropogenic activity (e.g., land use changes, pollution, and increased sedimentation) impact both the water quality of Lake Tanganyika directly and the lake's productivity via climate feedbacks (Ogutu-Ohwayo et al. 2016; Ivory et al. 2021; McGlue et al. 2021). Local fishermen similarly acknowledge spatial and temporal changes in fish abundance related to the ecological conditions of the lake (Bulengela et al. 2020; De Keyzer et al. 2020). Consequently, there is increasing recognition of the need to develop sustainable management strategies for the lake's pelagic fish stocks (Kimirei et al. 2008; Mölsä et al. 1999; Mölsä et al. 2002; Van der Knaap 2013; Van der Knaap et al. 2014; van Zwieten et al. 2002).

Lake Tanganyika's pelagic zone is relatively low in species diversity compared to that of other large African lakes: it consists predominantly of six endemic fish species belonging to two families (Coulter 1991). These include a monophyletic pair of mainly planktivorous clupeids (Stolothrissa tanganicae, Limnothrissa miodon) and four mainly piscivorous latids of the genus Lates (L. stappersii, L. mariae, L. microlepis, L. angustifrons). While all four Lates species likely have planktivorous larvae (van Zwieten et al. 2016), only L. stappersii remains pelagic throughout its life (Ellis 1978). The other three species move inshore to littoral weed beds as juveniles $(>3 \mathrm{~cm})$ before $L$. microlepis returns to the pelagic, $L$. mariae transitions to the deepwater benthic zone, and $L$. angustifrons can be found throughout the water column as adults (Coulter 1991). Of the four Lates species, L. stappersii is the most abundant and an important component of the current fishery of Lake Tanganyika (Coulter 1976, 1991; Kimirei et al. 2008; Mannini 1998a, b; Mölsä et al. 1999; Mölsä et al. 2002; Munyandero 2002; 
116 Sarvala et al. 2002; Van der Knaap 2013; Van der Knaap et al. 2014; van Zwieten et al. 117 2002).

118 The fish of Lake Tanganyika are influenced by a complex interplay of bottom-up control via changes in the supply of nutrient-rich deep waters, driven by different mixing regimes and upwelling rates (Bergamino et al. 2010; O'Reilly et al. 2003; Stenuite et al. 2007; Verburg \& Hecky 2009; Verburg et al. 2003), and top-down control via predators and fishing (Kimirei et al. 2008; Mannini 1998a; Mannini et al. 1996; Munyandero 2002; Van der Knaap 2013; Van der Knaap et al. 2014; van Zwieten et al. 2002). If spatially heterogeneous environmental factors, such as mixing regimes and nutrient supply, are consistent over generations and coupled with limited gene flow between spatially segregated populations, divergent selection on life history traits (e.g. spawning phenology, developmental timing and recruitment success) of fish in different parts of the lake could develop and persist. Spatial environmental variation in Lake Tanganyika, in combination with its large geographic extent, might therefore generate intraspecific genetic differentiation among populations of pelagic fish, as has been observed in pelagic cichlids in both Lake Tanganyika and Lake Malawi (Genner et al. 2010; Koblmüller et al. 2019). In these cichlids, strong spawning site fidelity (Genner et al. 2010) and differences in foraging behavior (Koblmüller et al. 2019) influence spatial genetic structure. In contrast, recent studies have found that the two pelagic clupeids in Lake Tanganyika show no evidence of spatial genetic structure (Junker et al. 2020; De Keyzer et al. 2019; Kmentová et al. 2020). Intraspecific spatial heterogeneity in spawning times (Coulter 1976; Ellis 1978), dominant diet items (Mannini et al. 1999), sizes at maturity (Mannini et al. 1996), and catch rates (Coulter 1991), along the length of Lake Tanganyika support the possibility that gene flow may be reduced among fish in different parts of the lake. Indeed, in L. stappersii, a study based on RAPD markers found individuals from the Kigoma region to be genetically differentiated from the rest of the sites sampled (Kuusipalo 1999), with some additional differentiation observed among individuals collected during the dry versus rainy season.

Here, we test these predictions using genomic data collected from each of the four Lates species from across their distribution within Lake Tanganyika. We use reducedrepresentation genomic sequencing datasets from four endemic Lates species- $L$. stappersii, L. mariae, L. microlepis, and L. angustifrons-to examine their genetic connectivity. We first assessed whether phenotypic identification coincides with genetically based species boundaries and then examined the extent to which each of the four species is genetically structured, as well as how the genetic structure observed in each species corresponds to its known life history and the environment. Based on known differences in life history among the four species, we hypothesized that we would find little genetic population 
expected the highest genetic differentiation in populations of the predominantly benthic $L$. mariae. Finally, we hypothesized that $L$. angustifrons would show intermediate amounts of structure since there is no evidence for large scale movements of this species, even though they are found throughout the water column.

\section{Material and Methods}

\section{Study system and sampling}

Between 2001 and 2019, we collected tissue samples (fin clips) from L. stappersii, L. mariae, L. microlepis, and L. angustifrons at 9 general locations along the Tanzanian shore of Lake Tanganyika, which together span the $\sim 490 \mathrm{~km}$ shoreline from the northern to southern border (Fig 1). All fish collections were made in partnership with researchers of the Tanzanian Fisheries Research Institute (TAFIRI) in Kigoma, Tanzania; most of the fish were obtained opportunistically from fishermen, and therefore were already dead when we received them. Fishermen generally fish within a $\sim 20 \mathrm{~km}$ radius from their landing sites, but there remains inherent uncertainty in the exact location and depth at which these fish were collected. Fish were identified in the field by sampling personnel following United Nations Food and Agricultural Organization (FAO) identification guidelines (Eccles 1992) and guidance from Tanzanian researchers and local fishermen, and genetic methods were later used to confirm species identities for each fish. The majority of fish were collected during two targeted sampling campaigns in 2017 and 2018, the first of which occurred at the end of the dry season (September-October 2017), while the second occurred just following the rainy season (April-May 2018). For fish collected from 2016-2019, we recorded length and weight, and took standardized pictures of each fish. For the few live fish that were caught as part of other research, we took cuvette photographs of the live fish and subsequently euthanized the fish with an overdose of MS222. We then took fin clips for genetic analysis from all fish. Specimens smaller than $600 \mathrm{~mm}$ were preserved in formaldehyde and archived in the collections at TAFIRI (Kigoma, Tanzania), EAWAG (Kastanienbaum, Switzerland), the University of Wyoming Museum of Vertebrates (Laramie, WY, USA), or the Cornell University Museum of Vertebrates (Ithaca, NY, USA). 


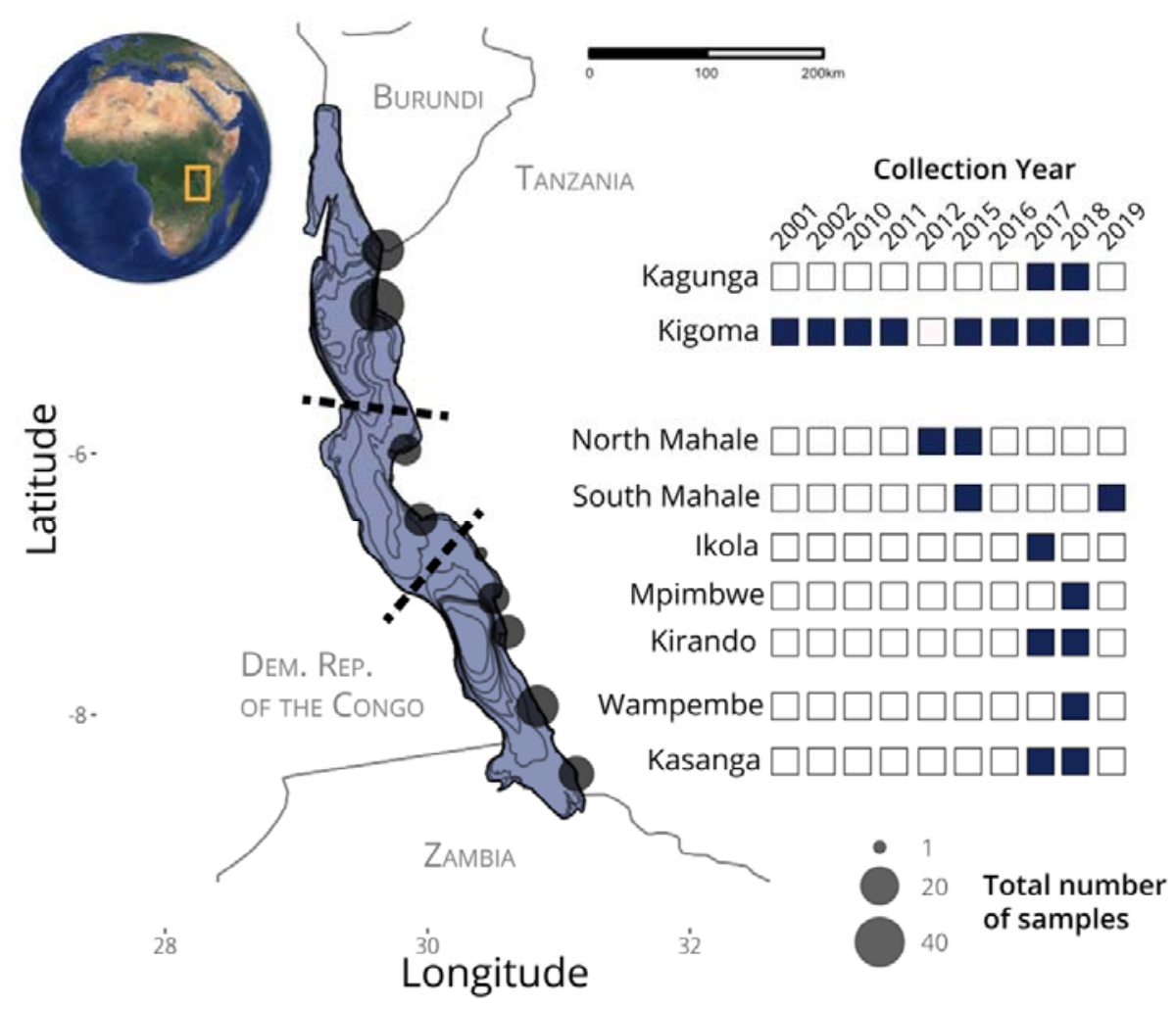

Figure 1. Sampling site locations and years in which fish were collected at each location (shaded boxes). Circle sizes on the map are proportional to the number of samples in the final dataset for all species combined, with a minimum of 1 and maximum of 45 samples collected per site across all years.

We extracted DNA from fin clips using DNeasy Blood and Tissue Kits (Qiagen, Inc.) following the standard protocol, with the addition of an RNAse A incubation step. We then prepared genomic libraries for a combination of genotyping-by-sequencing (GBS, for $L$. stappersii, $L$. mariae, L. microlepis, L. angustifrons) and restriction site associated DNA sequencing (RAD, for L. stappersii).

We prepared GBS libraries following protocols outlined in Parchman et al. (2012), using Msel and EcoRI restriction enzymes. Following fragmentation via restriction enzyme digestion, fragmented DNA was barcoded by ligating short, unique individual-identifying DNA fragments (barcodes) to each individual's fragmented DNA. These barcoded fragments were then amplified by PCR and pooled for sequencing. We ran two replicate PCRs for each individual and pooled the final PCR products into two libraries. The prepared libraries were sizeselected for 200-350bp fragments using Blue Pippin (Sage Science, MA). One library was 
202

203

204

205

206

207

208

209

210

211

212

213

214

215

216

217

218

219

220

221

222

223

224

225

226

227

228

229

230

231

232

233

234

235

at Austin's Genome Sequencing and Analysis Facility (UT GSAF; Austin, TX) and the other library was sequenced on one lane of Illumina HiSeq4000 (150bp, single-end) at the University of Oregon's Genomics and Cell Characterization Core Facility (Eugene, OR). Each library contained 192 individuals, and select individuals were duplicated across libraries to check for library compatibility.

The RAD libraries containing the L. stappersii were prepared for sequencing following the protocol by Baird et al. (2008) with the following modifications: we used between 400ng and 1000ng genomic DNA per sample and digested with Sbfl overnight. We tagged each individual using P1 adapters (synthesized by Microsynth) with custom six to eight base pair barcodes and multiplexed 67 barcoded individuals per library. The libraries were sheared using an S220 series Adaptive Focused Acoustic (AFA) ultra-sonicator (Covaris) with the manufacturer's settings for a 400 bp mean fragment size. We size-selected for fragments between 300 and 700bp using a sageElf (Sage Scientific Electrophoretic Lateral Fractionator; Sage Science, Beverly, MA). The enrichment step was done in 6 aliquots with a total volume of $200 \mu \mathrm{l}$. Volumes were combined prior to the final size selection (again 300 700bp) step using the sageELF. Sequencing was done by the Lausanne Genomic Technologies sequencing facilities (University of Lausanne, Switzerland). All three libraries were single-end sequenced on one lane each of the lllumina HiSeq2000 (100bp SE).

\section{Sequence data preparation}

We filtered raw sequencing reads from each library by first removing the reads derived from the PhiX genome and other common contaminants (e.g. excess barcodes, primers, and adapters) using bowtie2 (v2.0.0, Langmead \& Salzberg 2012). We filtered reads for an intact enzyme restriction site (Sbfl for RAD libraries, Msel and EcoRI for GBS libraries), demultiplexed the fastq files, and matched sequence barcodes to individual fish using custom perl and bash scripts.

For the GBS libraries, the reads were mapped to the chromosome-level Lates calcarifer reference genome (v3, NCBI Genome Assembly GCA_001640805.1; Vij et al. 2016) using bwa mem (v0.7.17; Li \& Durbin 2009) with default settings. Following alignment, we excluded any individual with $<50,000$ reads or less than $60 \%$ of raw reads assembled to the reference genome. We identified variable sites (i.e. single nucleotide polymorphisms; SNPs) in the assembly using SAMtools mpileup (v1.8; Li et al. 2009) and bcftools (v1.8; Li et al. 2009). In calling variable sites, we omitted indels and kept only high-quality biallelic variant sites (QUAL > 20 and $G Q>9$ ). We then filtered SNPs by minor allele frequency and amount of 
236 missing data using vcftools (Danecek et al. 2011), additionally only calling genotypes with a

237 minimum read depth of 5 . We then selected only SNPs with a minor allele frequency greater

238 than 0.01 that were present in at least $50 \%$ of individuals. Finally, we thinned each data set

239 to retain one SNP per locus (-thin 90). We assessed the distribution of missing data across

240 individuals and mean read depth across sites using vcftools (Danecek et al. 2011),

241 additionally using whoa (v0.0.2.999, Anderson 2018) in R (v4.0.4, R Core Team 2021) to

242 assess heterozygote miscall rate at low read depth sites to ensure that our read depth filter

243 was stringent enough.

244 For the RAD libraries, we barcode-trimmed reads down to 84 nucleotides using 245 process_radtags from Stacks (Catchen et al. 2013). The FASTX-toolkit v.0.0.13

246 (http://hannonlab.cshl.edu/fastx toolkit/) was used for quality filtering. In a first step, we kept

247 only reads with all base quality scores greater than 10; in a second step, we removed all 248 reads with more than $5 \%$ of the bases with quality score below 30 . The RAD library was then 249 mapped to the Lates calcarifer reference genome (Vij et al. 2016) and filtered, following the 250 same steps as for the GBS libraries above (MAF $>0.01$, missing data $<0.5$, one SNP per 251 locus, read depth $>5$ ). There was no overlap of individuals included in the RAD and GBS 252 sequencing libraries.

253 After filtering variants, we calculated the distribution of minor allele reads at heterozygous 254 sites for each individual in both the RAD and GBS libraries, using a custom bash script 255 (available at http://github.com/jessicarick/lates-popgen). If there is an excess of sequencing 256 errors or contamination for an individual, then we would expect the ratio of minor allele to 257 major allele reads to be significantly less than 1:1. Thus, we plotted the distribution of minor 258 allele reads at heterozygous sites for each individual and removed individuals with a 259 deficiency in minor allele reads across the majority of heterozygous sites. After removing 260 these individuals, we then re-called genotypes and re-filtered the SNP data set to produce 261 our final SNP data set.

Not all samples could be identified to species in the field. Some fish were caught deep and brought quickly to the surface by the fishermen, making them difficult to identify. Furthermore, juveniles of the large species L. microlepis, L. mariae and L. angustifrons can be difficult to distinguish. We used three different lines of evidence to confirm species identification and ensure that morphological identification matched genetic assignment: (1) phenotypic identification based on FAO species descriptions; (2) individual ancestry 

groups in phylogenetic analysis. The details for each of these steps are described below.

272 With the GBS data, we first visualized clusters in our data using principal component analysis using EMU (Meisner et al. 2021). EMU uses an iterative method to infer population structure in the presence of missing data and is therefore able to infer population structure even for datasets with high, uneven, or non-random proportions of missing data, which tend to produce bias in other PCA methods (Meisner et al. 2021). We used the EM acceleration method in EMU, using 10 eigenvectors for optimization (-e 10) with a maximum of 100 optimization iterations and keeping all eigenvectors as output. We then inferred species groups using the model-based genetic clustering program entropy (Gompert et al. 2014; Shastry et al. 2021). Entropy infers population structure from multilocus SNP data by assigning individuals partially or completely to groups using Bayesian estimation from genotype likelihoods. In taking uncertainty about individual genotypes into account via genotype likelihoods, the model integrates outcomes over genotype uncertainty. We converted our VCF containing sites with $<50 \%$ missing data to the mpgl genotype likelihood format using the vcf2mpgl R script (available at http://github.com/jessicarick/lates-popgen; adapted from https://bitbucket.org/buerklelab/mixedploidy-entropy/src, v2.0). We then ran entropy for $\mathrm{K}=4$ clusters, running three independent MCMC chains of 80,000 total steps, discarding the first 10,000 steps as burn-in, and retaining every $10^{\text {th }}$ value (thin=10), resulting in 7000 samples from the posterior distribution of each chain. Our MCMC chains were run in parallel using GNU parallel (Tange 2011). We checked MCMC chains for mixing and convergence of parameter estimates by plotting a trace of the MCMC steps. We then visualized assignments in $\mathrm{R}$ and assigned individuals to species based on group assignment probabilities (q).

We further corroborated species identities using maximum likelihood phylogenetic inference methods in RAxML (v8.1.17; Stamatakis 2014). We concatenated all SNPs, removed invariant sites using the raxml_ascbias python script (v1.0, from https://github.com/btmartin721/raxml ascbias), and used the Lewis correction for invariant sites with the ASC_GTRGAMMA model of molecular evolution (Lewis 2001) within RAxML to infer the maximum likelihood phylogeny, including L. calcarifer (Vij et al. 2016) as the outgroup. We used 100 rapid bootstraps to estimate confidence in our maximum likelihood tree. We then visually identified monophyletic groups on our maximum likelihood tree and used these groupings to further verify species identities from entropy and PCA clusters. 
305 We calculated genetic diversity for each species in ANGSD (v0.931, Korneliussen et al. 2014). From the BAM alignment files, we first calculated the site allele frequency likelihoods based on individual genotype likelihoods (option -doSaf 1) using the samtools model (option GL 1), with major and minor alleles inferred from genotype likelihoods (option -doMajorMinor 1) and allele frequencies estimated according to the major allele (option -doMaf 2). We filtered sites for a minimum read depth of 10 , minimum mapping quality of 20 , and minimum quality ( $q$-score) of 20 . From the site allele frequency spectra, we then calculated the maximum-likelihood estimate of the folded site frequency spectra (SFS) using the ANGSD realSFS program (with option -fold 1). The folded SFS were then used to calculate genomewide genetic diversity (Watterson's $\theta$ ), using the ANGSD thetaStat program (Korneliussen et al. 2013).

For each species, we then re-called variants to create a species-specific set of SNPs from the GBS data. As with the species combined GBS data, we identified variable sites using SAMtools mpileup and bcftools, omitting indels and keeping only high-quality biallelic variant sites (QUAL > 20 and $G Q>9$ ). We then filtered SNPs by minor allele frequency $(>0.01)$ and amount of missing data ( $<50 \%$ missing) using vcftools (Danecek et al. 2011), additionally only calling genotypes with a minimum read depth of 5 . Finally, we thinned to one SNP per locus (-thin 90).

For each species, we then performed principal component analysis (PCA) using EMU (Meisner et al. 2021), after using PLINK (v1.90b6.9, Purcell et al. 2007; Chang et al. 2015) to convert our VCF into BED format. EMU is able to infer population structure even for datasets with high, uneven, or non-random rates of missing data, which tend to produce bias in other PCA methods (Meisner et al. 2021). We again used the EM acceleration method, using 10 eigenvectors for optimization (-e 10) with a maximum of 100 optimization iterations and keeping all eigenvectors as output. We also performed PCA in EMU on the RAD dataset for L. stappersii.

We estimated heterozygosity at each site using the dartR package in R (v1.9.9, Gruber et al. 2018). We then used the Reich-Patterson $F_{S T}$ estimator (Reich et al. 2009), which is consistent even with small sample sizes, to calculate genetic divergence between sampling sites within each species. We calculated Reich-Patterson $F_{S T}$ estimates using a custom function in $\mathrm{R}$ (available at http://github.com/jessicarick/reich-fst), using functions from the 
our VCF. We then tested for isolation-by-distance (IBD) using a Mantel test (mantel.randtest from ade4 in R; v1.7-16, Dray \& Dufour 2007) to calculate the correlation between the natural logarithm of Euclidean geographic distance and standardized genetic distance $\left(F_{S T} / 1-F_{S T}\right)$ for all pairwise combinations of sampling sites within each species. We chose to pool these tests by sampling site, as we do not have more specific locations for where most of the fish were caught.

To test for intraspecific population subdivision more formally, we again used entropy. For each species, we ran entropy for $K=1$ to $K=6$ to infer the most likely number of ancestral groups within each species, and to assign individuals to these clusters. We ran three independent MCMC chains of 100,000 total steps, discarding the first 10,000 steps as burnin, and retaining every $10^{\text {th }}$ value (thin=10), resulting in 9000 samples from the posterior distribution of each chain. We checked MCMC chains for mixing and convergence of parameter estimates by plotting a trace of the MCMC steps. We then calculated the deviance information criterion (DIC) for each value of $\mathrm{K}$ and used the model with the lowest DIC as our best for modeling the variation observed in our data. From our entropy results, we assigned individuals to intraspecific groups using a cutoff of $q>0.6$ at each value of $K$. We then calculated the mean of Reich-Patterson $\mathrm{F}_{\mathrm{ST}}$ estimates between these $\mathrm{K}$ groups to understand how genetically distinct these groups are from one another. We also ran entropy at $\mathrm{K}=2$ and $\mathrm{K}=3$ using datasets with a more stringent missing data filter (miss $=0.9$ ) to test whether group assignments were contingent on the choice of bioinformatic filters. We also calculated ReichPaterson $F_{S T}$ estimates between each pair of individuals within each species in $R$, to facilitate comparisons that do not rely on group assignments. With the group assignments and individual pairwise $F_{S T}$ estimates, we statistically tested and graphically visualized relationships between these genetic measures and sampling year, sampling time of year, fish standard length, and juvenile status, to examine hypotheses about heterogeneity in spawning location and timing.

\section{Results}

The GBS libraries yielded an average of 245 million reads across 200 individuals. The RAD libraries containing the L. stappersii individuals yielded an average of 271 million reads, including 2.5\% - 8.6\% bacteriophage PhiX genomic DNA. On average, the mapping rate for L. stappersii individuals' GBS reads to the $L$. calcalifer reference genome was $89.8 \%$ (RAD, $89.6 \%$, and mapping rates averaged $91.6 \%$ for L. microlepis, $91.3 \%$ for L. mariae and $88.7 \%$ for $L$. angustifrons individuals. 
373 These mapping rates resulted in 1,463,702 unfiltered variable sites in L. stappersii,

$3741,286,810$ in L. microlepis, 961,153 in L. mariae and 764,456 in L. angustifrons. After filtering 375 for missing data $(<50 \%)$, minor allele frequency (MAF $>0.01)$, read depth ( $\operatorname{minDP}>5$ ), and 376 keeping only one SNP per locus (-thin 90), our species-specific GBS data sets contained 37738,160 SNPs from 58 L. stappersii individuals (RAD, 16,802 SNPs from 63 different 378 individuals), 33,429 SNPs from 34 L. microlepis samples, 30,431 SNPs from 38 L. mariae 379 samples and 19,519 SNPs from 20 L. angustifrons samples. The data set with all four 380 species contained 4,999,192 unfiltered and 44,823 filtered SNPs. None of the four species 381 exhibited heterozygote excess that would be expected if many sites were erroneously called 382 as heterozygous, and estimates of miscall rates are low for all read depths $>2$ (Fig. S1). The 383 majority of individuals who were lost were filtered out due to an imbalance in the reads 384 supporting major and minor alleles at heterozygous loci. In our final dataset, the mean read 385 depth was 15.2 for L. stappersii, 23.1 for L. microlepis, 20.9 for $L$. mariae, and 25.8 for $L$. 386 angustifrons (Fig. S2).

The species-combined SNP dataset for GBS data contained 200 individuals, of which we removed 50 individuals due to low numbers of reads $(n=2)$, low assembly rate to the reference genome $(n=4)$, or highly unbalanced read ratios at heterozygous sites $(n=44)$, which is indicative of contamination. The remaining individuals all had $>50,000$ reads that aligned to the $L$. calcarifer reference genome. The SNP alignment for RAxML contained 48,776 SNPs after removing invariant sites. The rooted maximum likelihood phylogeny (rooted on L. calcarifer; Fig. 2A) shows that each of the four species is reciprocally monophyletic. In addition, we infer a sister taxon relationship between $L$. mariae and $L$. angustifrons, and a sister taxon relationship between $L$. stappersii and $L$. microlepis (conflicting with the best supported topology in Koblmüller et al. 2021); however, short internode distances between these splits and low bootstrap support for the initial split suggest that all three speciation events may have occurred within a short period, and more investigation of the divergence history of the clade is warranted but not the focus of this current work. The PCA based on the GBS data was consistent with the species affinities indicated by monophyletic groups in RAxML (Fig. 2). The first genetic PC axis $(27.3 \%)$ separates $L$. stappersii from the remaining three species. The second PC axis (12.4\%) then separates $L$. mariae and $L$. microlepis from one another, and the third axis (4.8\%) separates $L$. angustifrons from the other three species. Using entropy at $\mathrm{K}=4$, we found each of the four species to make a distinct genetic cluster (Fig. 2C). Based on these combined analyses, 58 
angustifrons. While the genetic approaches revealed consistent species identifications, we had phenotypically misidentified one L. microlepis, one L. mariae and three L. angustifrons (Table S1).

\section{.}

(A)

Figure 2. Relationships among the four endemic Lates species in Lake Tanganyika based on the GBS data set, as demonstrated by their (a) phylogenetic relationships, (b) relationships using principal component analysis, (c) genetic clustering using entropy at $K=4$. The phylogeny has been rooted using L. calcarifer as an outgroup and nodal bootstrap support is indicated for the main nodes separating the species. In (a), genetic diversity of the given species, as measured using Watterson's Theta $\left(\theta_{W}\right)$ in ANGSD, is indicated. Percentages in (b) indicate that amount of variance explained by the given principal component axis (axes 1-4 shown). In (c) each vertical bar represents an individual, and the colors represent the proportion of ancestry assigned to each of the four species. Individuals are colored by a posteriori genetic assignment of species, rather than phenotypic identification.

\section{Population structure}

All four species had low but similar levels of genetic diversity (overall $\theta_{W}=0.00222$ ), and genetic diversity was elevated in $L$. stappersii $\left(\theta_{W}=0.00230\right)$ compared to the three other species (L. microlepis, $\theta_{W}=0.00094 ;$ L. mariae, $\theta_{W}=0.00092 ;$ L. anguistifrons, $\theta_{W}=0.00064$; Fig 2A). Genetic diversity for the RAD dataset in L. stappersii was similar to that estimated for the GBS data (RAD $\theta_{W}=0.00223$ ). Reflecting the distinctiveness seen in the all-species PCA and phylogeny, $F_{S T}$ values are high between species (mean Reich-Patterson $F_{S T}=$ 0.607; Table S2). None of the four species shows a significant relationship between genetic and geographic distance, suggesting an overall lack of isolation by distance (Fig. S3; all Mantel test $p$-values $>0.2$ ).

The PCA for L. stappersii based on GBS data (Fig 3A) did not reveal any clear genetic clusters. We similarly did not see any clustering in the RAD data for L. stappersii (Fig. S4). In 
436 entropy, $K=1$ was the best fit for the GBS data according to DIC (Fig. 5A); however, at $K=2$, 437 some individuals from Kigoma, North Mahale and South Mahale form a distinct genetic 438 cluster, and at $\mathrm{K}=3$, groups appear that co-occur at all sampling sites. These groups at $\mathrm{K}=2$ 439 and $\mathrm{K}=3$ were largely but not completely stable (i.e., the same individuals were clustered 440 together) at a higher threshold for missing data (miss $=0.5$ vs. miss $=0.9$ had 18 individuals 441 switching groups at $\mathrm{K}=2$ and 10 individuals at $\mathrm{K}=3$; Fig. S7, S8). This weak genetic structure 442 (between-cluster $\mathrm{K}=2$ Reich-Patterson $\mathrm{F}_{\mathrm{ST}}=0.00205,95 \%$ bootstrap Cl: 0.00084-0.00346, 443 Table S3) was also partly reflected in $\mathrm{F}_{\text {ST }}$ values between sampling sites, where $\mathrm{F}_{\mathrm{ST}}$ values of 444 comparisons between individuals of South Mahale or North Mahale with samples from other 445 sites were elevated, albeit small ( $\leq 0.012$, Fig. $4 \mathrm{~A})$; however, only the $\mathrm{F}_{\mathrm{ST}}$ values between 446 North Mahale and each of Mpinbwe and Kagunga had 95\% bootstrap confidence intervals 447 not overlapping zero (Fig. S9A). The RAD data showed similar weak structure at $\mathrm{K}=2$ (Fig. 448 S5; between-cluster $F_{S T}=0.00146$, 95\% bootstrap Cl: 0.00087-0.00207), but these groups 449 were found sympatrically at all five sampling sites, similar to the groups at $K=3$ for the GBS 450 data. At $K=3, F_{S T}$ values between groups for the RAD data had bootstrap confidence 451 intervals overlapping zero (Table S2).

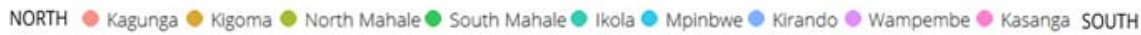

(A) Lates stappersii

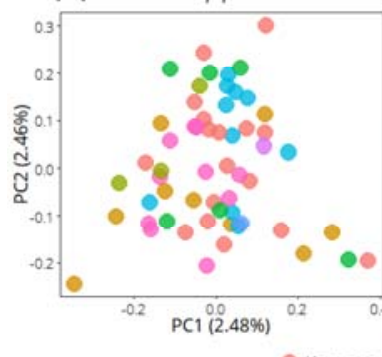

(B) Lates microlepis

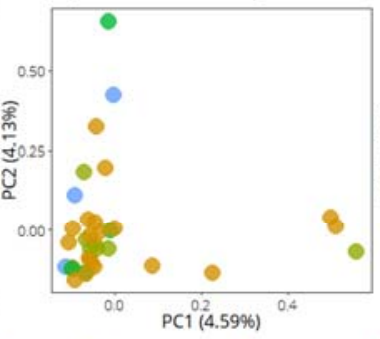

(C) Lates mariae

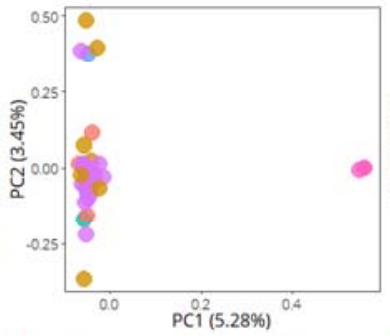

(D) Lates angustifrons

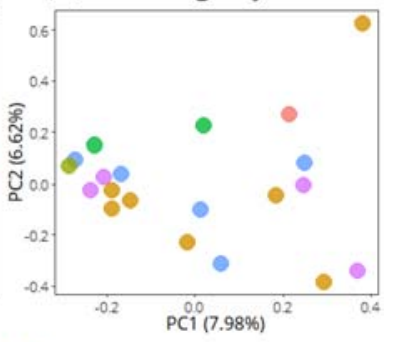

452

Kagunga $\bullet$ Kigoma $\bullet$ North Mahale $\bullet$ South Mahale $\bullet$ Ikola $\bullet$ Mpinbwe $\bullet$ Kirando $\bullet$ Wampembe $\bullet$ Kasanga

Figure 3. Principal component analysis of individual species based on the GBS data set, with colors indicating sampling locations, ordered from north to south. For each species, the plot shows the location of each individual on the two most dominant PCA axes, with percent variance explained in parentheses. 


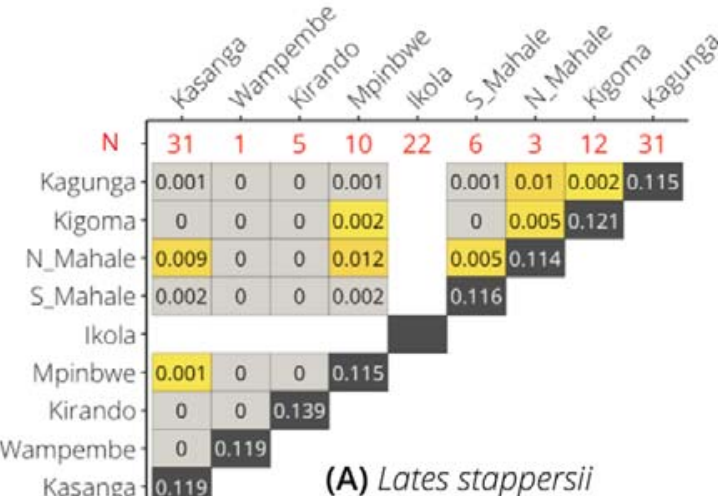

Kasanga 0.119

(A) Lates stappersii

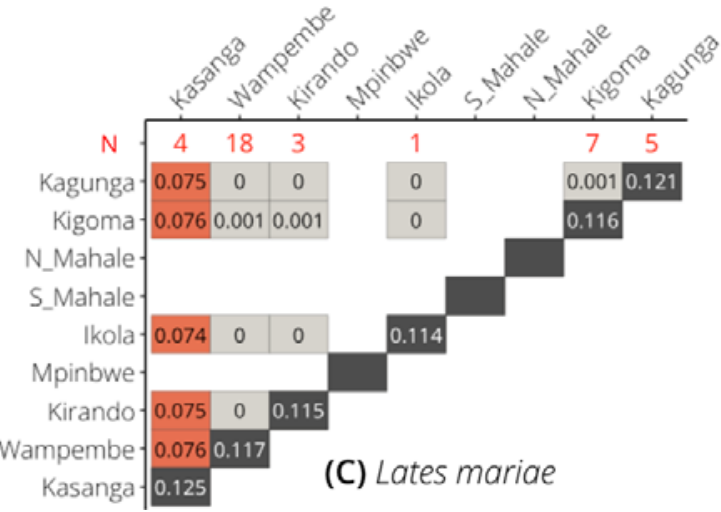

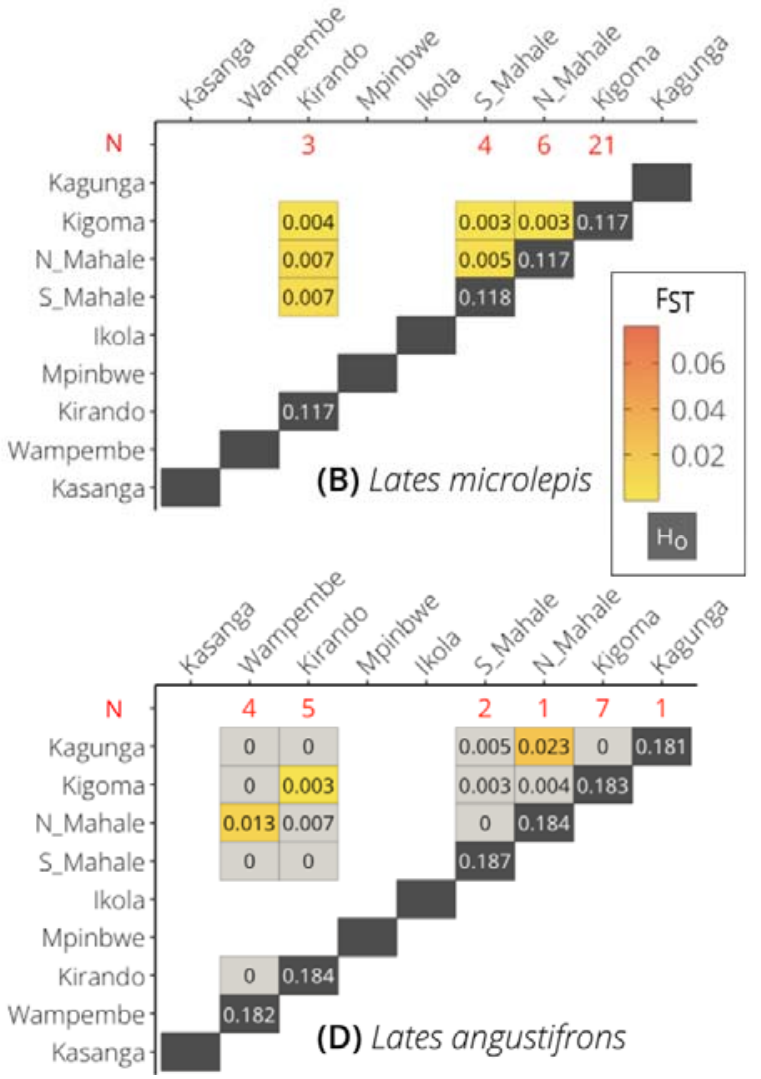

457

458

459

460

461

462

463

464

465

466

467

468

469

470

471

472

473

474

475

476

477

Figure 4. Heatmaps visualizing estimates of differentiation (Reich-Patterson $\mathrm{F}_{\mathrm{ST}}$ ) between sampling locations above the diagonal, with observed heterozygosities $\left(\mathrm{H}_{0}\right)$ along the diagonal (dark gray backgrounds). The red numbers across the top of each heatmap indicate sample size for the given species at the given sampling site. For $F_{S T}$ values, light gray indicates no differentiation (i.e., bootstrap confidence intervals overlap 0), yellow indicates low differentiation, and red indicates relative high levels of differentiation.

The PCA for L. microlepis did not reveal clear genetic clusters but it did highlight several individuals from the sampling sites Kigoma, North Mahale, and South Mahale, that were differentiated from the other samples on PC1 and PC2 (Fig. 3B). These differentiated individuals were not those with high amounts of missing data (Fig. S10B). All of the sampling sites had low differentiation (mean $\mathrm{F}_{\mathrm{ST}}=0.00471$; Fig. 4B) but all pairwise comparisons had 95\% confidence intervals that did not overlap zero except for the Kirando-Kigoma and Kirando-South Mahale comparisons (Fig. S9B). In entropy, $\mathrm{K}=1$ was found to be the best fit using DIC (Fig. 5B); at $\mathrm{K}=2$, four individuals collected in Kigoma are differentiated from the others, albeit with low genetic differentiation and a between-group $\mathrm{F}_{\mathrm{ST}}$ estimate that overlaps zero (Reich-Patterson $\mathrm{F}_{\mathrm{ST}}=0.00074,95 \% \mathrm{Cl}$ : $-0.00389-0.00549$; Table S3). At $\mathrm{K}=3$, the additional group is found at all four sampling sites; the differentiation between the two largest groups is low, but significant (Reich-Patterson $\mathrm{F}_{\mathrm{ST}}=0.0030,95 \% \mathrm{Cl}$ : $0.00155-0.00474$ ), while the $F_{S T}$ estimates between each of these groups and the smaller group identified at 
$\mathrm{K}=2$ had $95 \%$ bootstrap confidence intervals overlapping zero. These groups at $\mathrm{K}=2$ and $\mathrm{K}=3$ were largely stable at a higher threshold for missing data (miss $=0.5$ vs. miss $=0.9$ had 4 individuals switching groups at $\mathrm{K}=2$ and 8 individuals at $\mathrm{K}=3$; Fig. S7, S8).

The PCA for $L$. mariae clusters three individuals from Kasanga separate from all other individuals on the first PC axis (explaining $5.28 \%$ of the variance), while the second PC axis separates out some individuals from Kigoma, Wampembe, and Kirando from the others (Fig. 3C). The differentiation among individuals on both PC1 and PC2 does not correlate with the individuals' amount of missing data (Fig. S10C). In entropy, $K=2$ has the lowest DIC estimate, where the three individuals from Kasanga form a distinct cluster (Reich-Patterson $\mathrm{F}_{\mathrm{ST}}$ between entropy-identified groups $=0.119$, 95\% bootstrap Cl: 0.113-0.127; Fig 4C and Table S3). At $\mathrm{K}=3$, most of the other sampling sites have individuals assigned to both of the non-Kasanga clusters, although these two non-Kasanga clusters have very low differentiation $\left(\mathrm{F}_{\mathrm{ST}}=0.00359\right.$, 95\% bootstrap Cl: $\left.0.00180-0.00623\right)$ compared to that between the Kasanga cluster and each of the other two clusters (mean $F_{S T}=0.120$ ). These groups at $\mathrm{K}=2$ and $\mathrm{K}=3$ were largely stable at a higher threshold for missing data (miss $=0.5$ vs. miss $=0.9$ had no individuals switching groups at $\mathrm{K}=2$ and 15 individuals at $\mathrm{K}=3$; Fig. S7, S8). The $F_{S T}$ estimates between all fish sampled from Kasanga and other sampling sites are all significantly different from zero (Fig. S9C). All other between-site $\mathrm{F}_{\mathrm{ST}}$ estimates had 95\% bootstrap confidence intervals overlapping zero.

For L. angustifrons, the PCA did not reveal any genetic structure (Fig. 3D) and the clustering model with $\mathrm{K}=1$ is the best fit for our data (Fig. 5D). Clusters identified at higher levels of $\mathrm{K}$ had low differentiation (Table S3; $\mathrm{K}=2, \mathrm{~F}_{\mathrm{ST}}=0.00498 ; \mathrm{K}=3$, mean $\mathrm{F}_{\mathrm{ST}}=0.0228$ ), and were found co-occurring at most sampling sites (Fig. 5D). These groups at $\mathrm{K}=2$ and $\mathrm{K}=3$ were largely but not completely stable at a higher threshold for missing data (miss $=0.5$ vs. miss $=0.9$ had 4 individuals switching groups at $\mathrm{K}=2$ and 7 individuals at $\mathrm{K}=3$; Fig. S7, S8). In addition, only three pairwise $F_{S T}$ estimates between sampling sites were significant: Kagunga-North Mahale, Kigoma-Kirando, and North Mahale-Wampembe (Fig. S9D); however, even these sites had low differentiation (mean $F_{S T}$ between sites $=0.0132$ ), and it should be noted that North Mahale only has one fish sampled, so these estimates may not be indicative of patterns at the site as a whole. 


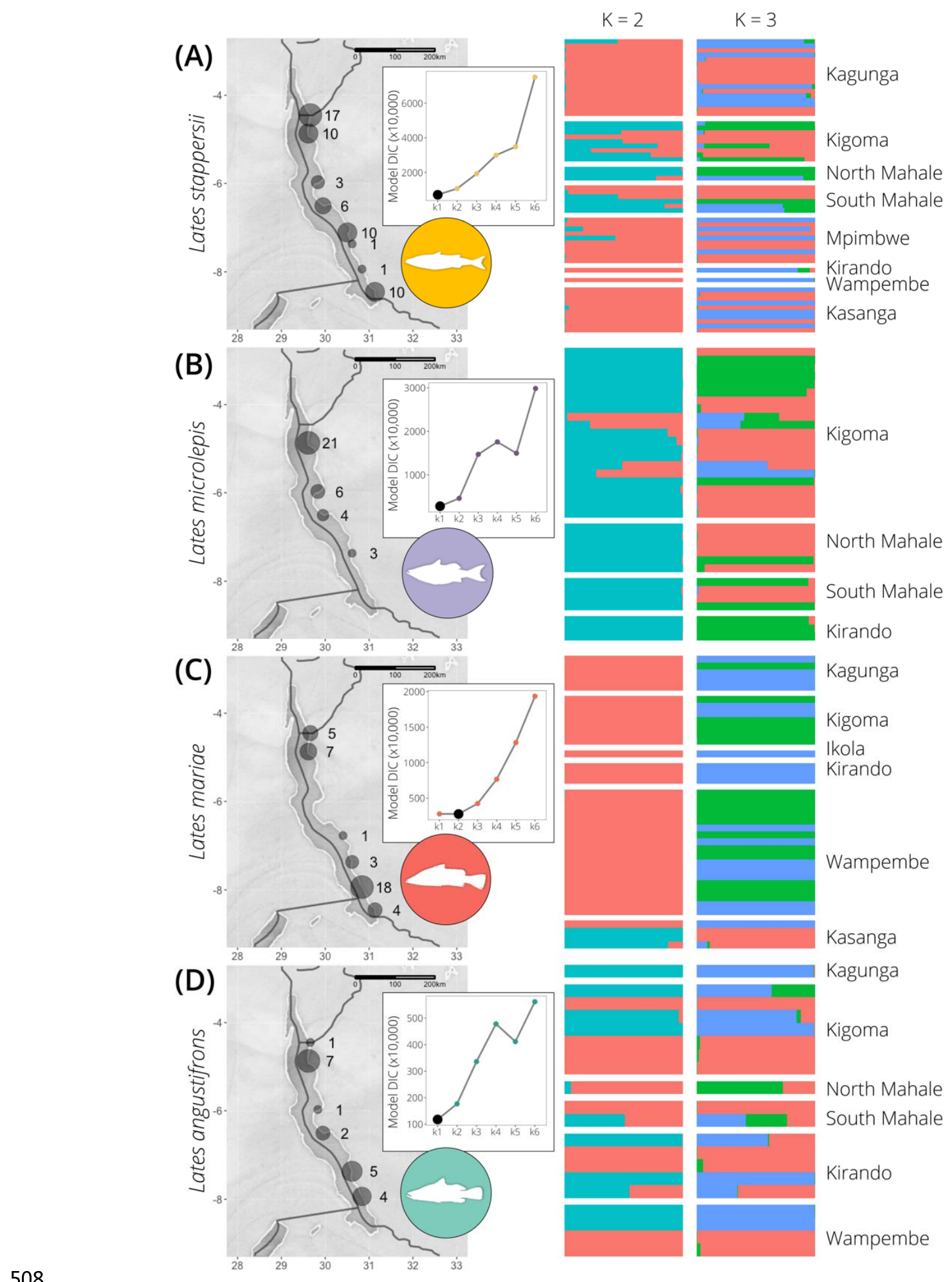

Figure 5. Intraspecific population structure inferred from the GBS data set and entropy analyses at $510 \quad \mathrm{~K}=2$ and $\mathrm{K}=3$ for (A) L. stappersii, (B) L. microlepis, (C) L. mariae, and (D) L. angustifrons. Maps show 511 the distribution of individuals among sampling locations and inset plots show discriminant information 512 content (DIC) values for each value of $\mathrm{K}$, with the lowest DIC value for each species indicated with a 513 larger black dot. In colored barplots, each bar corresponds to one individual and colors indicate 514 inferred ancestry from each of the $K$ groups. Plots for values of $K=4$ to $K=6$ can be found in 515 Supplementary Fig. S6. 
517 We find no relationship between size difference and extent of differentiation (pairwise $\mathrm{F}_{\mathrm{ST}}$ or 518 genetic cluster membership) in L. mariae, L. microlepis, or L. angustifrons. In L. stappersii, a weak positive relationship exists between the difference in standard length and the extent of genetic differentiation $\left(\mathrm{F}_{\mathrm{ST}}\right)$ between individuals, such that individuals that are more different in size are also more differentiated genetically (Fig. S12). In L. microlepis, we found no relationship between genetic cluster membership and juvenile status at $\mathrm{K}=2$ or $\mathrm{K}=3$ when examining only the fish collected in Kigoma, the only site with large enough numbers for doing this analysis. We additionally find no systematic difference between $F_{S T}$ estimates between only juvenile fish and those estimated between only adult fish for $L$. microlepis or $L$. mariae (Fig. S13, S14). When only looking at fish collected the same site (Fig. S14), we find that juvenile-adult comparisons had higher $F_{S T}$ values in $L$. mariae than adult-adult comparisons (two-tailed t-test: $\mathrm{t}=2.3484, \mathrm{df}=28$, $\mathrm{p}$-value $=0.02615$ ), but did not differ from juvenile-juvenile comparisons (two-tailed t-test: $t=-1.7863$, $\mathrm{df}=31.781, \mathrm{p}$-value $=0.0836$ ), and juvenile-juvenile and adult-adult comparisons were not significantly different from one another (two-tailed t-test: $\mathrm{t}=1.9658$, $\mathrm{df}=151, \mathrm{p}$-value $=0.05116$ ). In $\mathrm{L}$. angustifrons, $\mathrm{F}_{\mathrm{ST}}$ values among juveniles at the same site were lower than those between juveniles and adults at the same site (two-tailed t-test: $t=-3.8334, \mathrm{df}=16.646, \mathrm{p}$-value $=0.001376$ ) or between adults at the same site (two-tailed t-test: $t=2.2254, d f=27.295, p$-value $=0.0345$ ), while $F_{S T}$ estimates did not differ significantly between juvenile-juvenile and adult-adult comparisons (two-tailed t-test: $\mathrm{t}=-1.6493, \mathrm{df}=14.084, \mathrm{p}$-value $=0.1212$ ). In $\mathrm{L}$. microlepis, $\mathrm{F}_{\mathrm{ST}}$ estimates for fish from the same site were marginally higher in juvenile-juvenile pairs than in adult-adult pairs (two-tailed t-test: $t=2.2525, \mathrm{df}=19.005$, p-value $=0.03631$ ), but did not differ between each of these categories and juvenile-adult comparisons ( $p$-value $>0.07$ ). We do not find any evidence for juveniles collected at the same time of year to be more closely related than juveniles collected during different parts of the year in $L$. microlepis (two-tailed t-test: $t=-$

542 1.2229, $\mathrm{df}=378.64, \mathrm{p}$-value $=0.2221$; Fig. S15), and do not have enough data to analyze

543 this relationship in the other three species.

\section{Discussion}

546 Differences in life history characteristics can have significant influence on differences in 547 population genetic structure among taxa, even in closely related species. In this study, we 548 analyzed population genetic structure within the four endemic pelagic top predators of Lake 549 Tanganyika, Lates spp., using reduced-representation genomic sequencing data sets. From 550 our samples collected at discrete sampling sites along the length of the lake, we find three 
551 main patterns: first, we find a strongly differentiated genetic group in Lates mariae at the

552 most southern sampling site, Kasanga; second, we find no additional pattern of isolation-by-

553 distance or geographic population structure within any of the four Lates species; and third,

554 we find weak evidence for weakly differentiated genetic groups that exist in sympatry at the

555 majority of sites in all four species. While numerous explanations could account for a lack of

556 geographic population structure and the existence of sympatric weakly differentiated groups,

557 more research is needed to understand mechanisms causing the population structure

558 identified in the different species. These results have potential implications for understanding

559 the conservation and management of these fishes, with implications for this regionally

560 important fishery.

561

\section{Strong genetic differentiation in southern Lates mariae}

563 In L. mariae, the majority of samples from the most southern site, Kasanga, are genetically

564 differentiated from all other samples. The magnitude of $F_{S T}$ estimates between these distinct

565 individuals and the other $L$. mariae individuals $\left(F_{S T} \sim 0.135\right)$, suggests unexpectedly strong

566 intraspecific genetic structure given a lack of obvious geographic barriers. Unfortunately, our

567 sampling does not include individuals from this furthest south site for $L$. microlepis or $L$.

568 angustifrons, so we cannot determine whether this pattern is also reflected in the other two

569 large Lates species.

570 It is plausible that the differentiation in $L$. mariae may be driven by known limnological

571 patterns that differ between the northern and southern portions of the lake. In particular, the

572 southern part of the lake experiences intense upwelling of cold, nutrient rich waters from the

573 deep during the dry season (Verburg et al. 2011), which mixes with oxygenated water from

574 the surface and consequently has high nutrient concentrations and primary productivity rates,

575 which in turn fuels zooplankton growth (Plisnier et al. 2009; Loiselle et al. 2014). In contrast,

576 the northern part of the lake has a more stable, persistently stratified, and clear water column

577 (Plisnier et al. 1999; Bergamino et al. 2010; Mziray et al. 2018). As a result, there is

578 asynchronous variability between environments in the north and south (i.e., higher primary

579 productivity in the south than in the north of the lake), as well as high environmental

580 variability over the course of the year in the south (i.e, higher primary productivity in the dry

581 season compared to the rainy season). Spatial variability in conditions affects zooplankton

582 community composition, with shrimps and calanoid copepods prevailing in the south (Kurki et

583 al. 1999), while cyclopoid copepods and medusa dominate in the north (Kurki et al. 1999;

584 Cirhuza and Plisnier 2016). Increases in primary productivity generally lead to high densities

585 of zooplankton, as well as Stolothrissa and Limnothrissa sardines (Mulimbwa et al. 2014), as

586 the blooms seem to positively affect sardine spawning and recruitment, although recruitment 
587 pulses sometimes occur during times of poor nutrient conditions as well. Mannini et al. 588 (1999) found corresponding spatial variability in diet for L. stappersii, but spatial variability in 589 diet has not been documented for L. mariae or the other large-bodied species. While adult $L$. 590 mariae do not depend on the pelagic sardines as a primary food source (Coulter 1976), it is 591 possible that such temporal nutrient variability leads to variability in $L$. mariae prey (benthic 592 cichlids) as well.

Such a spatially and temporally variable environment might lead to local differential selection 594 in the southern part of the lake, and this selection combined with restricted movement of 595 individuals between this and other populations of $L$. mariae could lead to the genetic 596 differentiation in individuals from Kasanga. L. mariae abundance is greater in the south than 597 in the north (Coulter 1970; I. Kimerei, E. Sweke, personal observation), which suggests that 598 spatial variation in environmental conditions may underlie differences in fitness or population 599 growth. A variable environment may also lead to within-site differentiation among individuals 600 caught in Kasanga if the two groups were to adapt to this variability in different ways. Another 601 possibility is that one group is adapted to remaining in Kasanga, while the other disperses 602 further throughout the lake. While all L. mariae individuals sampled from Kasanga were 603 mature and L. mariae is believed to be the least vagile of the four Lates species (Coulter 604 1976), it is not clear how vagile these individuals typically are; it is possible that some 605 individuals remain resident while others migrate, as is the case in Atlantic cod (Kirubakaran 606 et al. 2016; Berg et al. 2017). To investigate these possibilities, future studies should expand sampling in the southern portion of the lake, including sampling various life stages of this and other Lates to understand the genetic composition of the southern populations and any important ecological implications of genetic differentiation.

610 It is also possible that the differentiation in the most southern part of Lake Tanganyika in $L$. 611 mariae is related to the lake's bathymetry and differences in the depth of the oxygenated 612 layer, as suggested by Coulter (1976), who observed asynchronous changes in local 613 abundance between L. mariae populations in the southwest versus southeast arms of the 614 lake. From catch rates, Coulter (1970) observed that southern populations of at least two of 615 the Lates species, L. angusitfrons and L. mariae, were likely distinct, as they were heavily 616 exploited in the southeast arm with no apparent effect on catches in the southwest arm. 617 These southern reaches of the lake are relatively shallow, and the oxygenated layer extends 618 up to $200 \mathrm{~m}$ deep, which allows for a large area of oxygenated bottom to be available to 619 benthic species (Coulter 1976).

620 Despite the life history differences between pelagic cichlids and the Lates species, the 621 genetic patterns observed in L. mariae are similar to those observed previously in the 622 benthopelagic cichlids. For the four benthopelagic Diplotaxodon species of Lake Malawi with 
623 genetically structured populations, research by Genner et al. (2010) indicated spawning site

624 fidelity drives population structure. In contrast, Koblmüller et al. (2019) hypothesized that 625 preying upon benthic cichlids-which requires shorter-distance movements than preying on 626 pelagic prey-can explain the genetic structure in the Bathybatini of Lake Tanganyika. 627 Similar to the Bathybatini, L. mariae hunts benthic cichlids and is described as relatively 628 resident (Coulter 1976). However, our results did not recover any evidence for significant 629 isolation-by-distance in L. mariae, suggesting that the genetic structure that we observe is 630 likely not the result of dispersal limitation. However, females of $L$. mariae do aggregate over 631 specific spawning grounds (Coulter 1991), similar to Diplotaxon. If samples of L. mariae were 632 taken over spawning grounds, it is possible that results would reveal clearly distinct 633 populations as would be expected from strong spawning site fidelity and differentiation 634 between these stocks. However, the sampling methods used in our study (i.e., obtaining fish 635 from fishermen) limit our knowledge of whether fish were collected over spawning grounds. 636 In addition, these sampling methods mean that a range of ages and reproductive maturities 637 of fish were included in our study. To our knowledge, the location of spawning grounds has 638 not been documented for any of the four Lates species. In addition, it is unclear how the 639 pelagic phase of eggs, larvae, and small juveniles $(<2.5 \mathrm{~cm})$, of $L$. mariae influences dispersal 640 in the species, as spawning pelagically (Riginos et al. 2014) and having a long pelagic larval 641 stage (Selkoe \& Toonen 2011; Shanks 2009) generally facilitate gene flow and reduces 642 population structure in marine taxa (but see Bay et al. 2006; Riginos et al. 2011), although 643 these patterns are complicated by specific behavior exhibited by both adults and larvae. 644 Future studies in L. mariae should sample spawning fish and work to understand the location 645 of spawning grounds to further assess whether the intraspecific divergences that we observe 646 could arise from spawning site fidelity.

Although the samples spanned the $\sim 550 \mathrm{~km}$ Tanzanian shoreline of Lake Tanganyika, none of the genetic differences between populations relates to geographic distance, suggesting no isolation-by-distance in any of the four species. In addition, admixture models with only a single panmictic population fit our data the best in all species except for L. mariae (Fig. 5).

653 This suggests that individuals of all four species are mobile enough that gene flow is not 654 restricted among our sampling sites, other than the distinct Kasanga population.

655 Deviations from isolation-by-distance are common among marine animals, where 656 geographically proximate populations often have greater population differentiation than 657 populations further apart, a phenomenon sometimes referred to as "chaotic patchiness" 658 (Johnson and Black 1984). This phenomenon has been attributed to larval dispersal via 
ocean currents (White et al. 2010), and it is possible that currents within Lake Tanganyika may similarly disperse Lates eggs and larvae prior to their movement to inshore habitats. While it is presumed that all four Lates species spawn pelagically (Ellis 1978; Coulter 1991) and the larvae of the larger three species are planktonic until $\sim 3 \mathrm{~cm}$ in size (Coulter 1976), spawning behavior, spawning location within the lake, and the length of time that larvae may be carried around by lake currents remain unknown for all four species. Lake currents vary throughout the year (Plisnier et al. 1999; Verburg et al. 2011), and therefore understanding the timing and location of reproduction for all four species will be important to understanding the role that these currents may play in dispersing planktonic eggs and larvae.

While geographically widespread populations are not particularly surprising given the large body sizes and long-distance swimming capabilities of these fishes, including evidence for long-distance movements in L. stappersii and L. microlepis, it conflicts with evidence of a resident lifestyle in L. mariae (Chapman 1976; Coulter 1976, 1991; Muller et al. 2001) and evidence for some spatial structure in the Kigoma area in a previous population genetic study of L. stappersii (Kuusipalo 1999). It should also be noted that our sampling is unbalanced among sites and lacking in the most southern reaches of the lake for $L$. angustifrons and $L$. microlepis. It is therefore possible that more (albeit still likely weak) genetic structure exists than we have been able to detect with available samples. That being said, Koblmüller et al. (2019) similarly found no evidence of genetic population structure in two eupelagic bathybatine cichlid species of Lake Tanganyika, Koblmüller et al. (2015) found no evidence for poulation structure in the pelagic cichlid Boulengerochromis microlepis, and De Keyzer et al. (2019) and Junker et al. (2020) found no population structure in either of the pelagic sardine species. There are many life history differences between the sardines, cichlids, and Lates that occur sympatrically in the open waters of Lake Tanganyika, but these results together suggest that spatial environmental heterogeneity along the length of the lake is not sufficient to restrict gene flow and produce geographically isolated or clinal populations in these diverse species.

\section{Sympatric, weakly-differentiated groups exist in all four species}

While our admixture models suggest panmixia as the best model in $L$. stappersii, $L$. microlepis, and L. angustifrons, and two distinct groups in L. mariae, we find groups with restricted gene flow in all four Lates species that co-occur at the same sampling sites at higher levels of K. Model DIC values suggest that these higher levels of division are not the best fit for our data and differentiation is weak between groups (mean $F_{S T}=0.032$; Table S3), but individual assignments do not behave as we would expect if we were to increase the number of groups in a truly panmictic populations. In our data, we see individuals being 
695

696

697

698

699

700

701

702

703

704

705

706

707

708

709

710

711

712

713

714

715

716

717

718

719

720

721

722

723

724

725

726

assigned with high confidence to one group or another (i.e., each bar is mostly a single color in Fig. 5 and Fig. S6); if the population were truly panmictic, then we would expect to see all individuals showing genetic contributions from all groups. For that reason, we believe these weakly differentiated groups may be biologically relevant, albeit weakly differentiated. These groups do not appear to correspond to sex or any other phenotypic character that we recorded. There are several potential explanations for this consistent structure, of which we will explore the evidence for three: (a) spatially segregated spawning sites and philopatry in these species, where adults move throughout the lake but return to the same spawning grounds to breed; (b) temporally segregated spawning groups within these species; or (c) historical allopatric groups that have become sympatric yet partially retained their genetic distinctiveness.

Genetically distinct groups that occur in sympatry must have at least partial reproductive isolation, and in fish without clear sexual selection, this often occurs intraspecifically as a segregation of spawning either in space or time. If there is site fidelity to regionally differentiated spawning grounds but between-region movement outside of spawning, then this could create patterns of differentiation such as those that we observe. If spatially segregated spawning sites exist, then the adults that are sampled at a single site may have migrated from a more distant site (unless they are collected while spawning, in which case we would expect the genetics to match with their geographic location), but we would expect littoral juveniles to exhibit a stronger pattern of geographic-based differentiation or isolation by distance. In addition, we would expect juveniles collected at the same site to be more closely related than adults collected at the same site, or juveniles and adults collected at the same site. We did not find this to be the case in any of the four species (Fig. S14), with the caveat that our sample sizes for these comparisons is low and our data may exhibit batch effects due to our sampling strategies. We did find stronger differentiation in L. angustifrons and L. mariae between juveniles and adults collected at the same site than between juveniles and juveniles collected at the same site; however, the mean $F_{S T}$ for adult-adult comparisons was not larger than that of juvenile-juvenile comparisons, so the evidence supporting this hypothesis is mixed. However, again, our sample size in each of these categories for each sampling site is small, and therefore future studies would be helpful for quantifying support for this hypothesis.

Genetic differentiation related to temporal stratification in spawning behavior-such as having a population of "dry season" spawners and a population of "rainy season" spawnerscould also explain the existence of sympatric groups. Coulter (1976) suggested that the four species likely spawn continuously, but with peaks in number of ripe females from August to November/December, and potentially other smaller peaks in March/April. In addition, observations of well-differentiated size distributions of young Lates found in littoral weed 
732 beds suggests periodicity in spawning (Coulter 1976). Unfortunately, we do not have data on

733 the spawning status of the fish collected in this study, which would facilitate investigating this

734 hypothesis. However, we were able to test whether juveniles collected at a similar time of

735 year were more closely related than those collected at a different time of year, regardless of

736 sampling site. We do not find any evidence for juveniles collected at the same time of year to

737 be more closely related than juveniles collected during different parts of the year in $L$.

738 microlepis (Fig. S15); however, our sampling is not conducive to being able to rigorously

739 investigate this relationship, as some sites were only sampled in one year or at one time of

740 year. Additionally, only a few juveniles were collected opportunistically from each species, as

741 they are too small to be targeted by fishermen. Investigating the possibility of discrete

742 spawning groups will be an important next step in understanding the weak genetic structure

743 present in these species, and will require more targeted sampling.

744 Another third potential explanation for this consistent structure could lie in the geologic 745 history of Lake Tanganyika. With an estimated age of $9-12$ million years, Lake Tanganyika is 746 one of the oldest lakes in the world and has been influenced by extensive lake level 747 fluctuations (Cohen et al. 1993). Based on the bathymetry of the lake, it is thought that Lake 748 Tanganyika was divided into three paleo-lakes during periods of extremely low water levels 749 (Cohen et al. 1997). Environmental fluctuations such as these may have led to oscillations 750 between sympatry or parapatry and allopatry for fish populations within the lake, which have 751 been invoked as a "species pump" (Greenwood 1981; Sedano et al. 2010) contributing to 752 diversity in some clades in the littoral (Janzen \& Etienne 2017; Sturmbauer et al. 2016) and 753 benthic or pelagic (Duftner et al. 2005; Koblmüller et al. 2005) zones of the lake. While it has 754 recently been suggested that the origin of the Lake Tanganyika Lates radiation is much 755 younger than other endemic fish radiations in the lake (Koblmüller et al. 2021), it remains 756 unclear whether speciation in this clade was similarly triggered by these environmental 757 fluctuations - as well as whether more recent fluctuations could explain our contemporary 758 observations of weak genetic structure. Such fluctuations could also lead to persistent 759 genetic structure within species, for example, if spawning site fidelity within separate basins 760 is maintained after the basins reconnect. However, we would expect strong philopatry to 761 produce much stronger genetic differentiation than observed here.

762 Interestingly, L. stappersii in this study which were caught during the dry season in the south 763 of the lake show distinct carbon isotope signals from samples caught in the north (Ehrenfels 764 et al, in prep). These differences in carbon isotope ratios correlate with different rates of 765 primary productivity in the northern and the southern basin during the dry season (Loiselle et 766 al. 2014), and with differences in prey composition in different areas of the lake (Plisner et al. 767 2009; Kurki et al. 1999). This is evidence for at least partly resident populations of $L$. 768 stappersii, which stay in the northern or southern basin during the dry season for long 
enough to incorporate the different isotopic signals of these basins. Such movement patterns may reduce gene flow between $L$. stappersii populations from the basins; however, these isotopic groups do not seem to correspond to the genetic groups found at $\mathrm{K}=2$ here (Ehrenfels et al, in prep).

With these potential explanations in mind, it is important to keep the limitations of our data in mind. Admixture analyses using programs such as STRUCTURE (Pritchard et al. 2000) have been shown to be biased with unbalanced sampling among sites, such that differentiation is often overestimated at sites with the most individuals and underestimated at sites with few individuals (e.g., Puechmaille 2016; Wang 2017). While it is unclear whether this is true for entropy analyses as well, it is important to interpret our analyses with this in mind. For example, we find multiple groups at the Kigoma site in L. microlepis at $\mathrm{K}=2$, which is also the site with the most samples present in the data set (Fig. 5B). Furthermore, our assignment of individual locations to fisherman landing sites or markets is less precise than if we had exact locations where the fish were caught. While we only have general knowledge of how far fishermen generally travel from the landing site to fish during the day, it is possible that fish collected at the same sampling site were collected up to $\sim 40 \mathrm{~km}$ away from one another. In addition, our samples collected in the Kigoma fish markets may have come from farther away from those collected at the landing sites (Petit \& Shipton 2012). These limitations influence our ability to detect geographic trends in our data; however, we believe that these limitations would only have minor effects on the inferences discussed here.

\section{Recommendations for conservation and sustainable management of Lake Tanganika's Lates}

We find evidence for unexpected weak yet genetically distinct and sympatric groups within all four species. We furthermore find evidence for genetically distinct group within L. mariae at our most southern sampling site, in agreement with previous studies suggesting that this species is the most resident of all four Lates species (Coulter 1976, 1991). Intriguingly, this structure is not linked to isolation-by-distance, but rather involves differentiation between some individuals at multiple sites, suggesting the existence of a mechanism for maintaining differentiation while these distinct genetic groups are in sympatry. In contrast, the low $F_{S T}$ estimates between samples from distant sites and lack of isolation-by-distance suggest that these species do disperse over large distances, suggesting a need for management of these species on at a lake-wide scale. Furthermore, it is likely that more extensive sampling of these little-studied species may uncover important additional diversity.

To maintain intraspecific genetic variation, viable population sizes, and possible adaptive differences between genetic groups within the Lates species, more research is needed on 
804 the location and timing of spawning for the four species and whether restricting catches in

805 certain areas and periods of the year could help the larger-bodied species to recover from

806 low population sizes. Harvest restrictions have been implemented in regions around Lake

807 Tanganyika where community conservation areas are active (Kimirei \& Sweke 2018) but are 808 not in place throughout the lake. Such management practices are performed successfully in

809 the cod fishery of Denmark (albeit after collapse of this fishery and extinction of many of the 810 original stocks; Dahle et al. 2018), and in the salmon fishery of Bristol Bay (Schindler et al. 811 2015; Schindler et al. 2010). However, implementation of such a strategy requires detailed 812 evidence of spawning ground locations and timing of spawning, as well as for fishermen to 813 be able to distinguish between the genetically distinct stocks. Therefore, studies are needed 814 to shed more light on the phenotypic, ecological, and phenological characteristics of the 815 genetic groups within each species. In addition, studies are needed to examine whether fish 816 from different spawning grounds form the distinct genetic clusters that we identified in this 817 study. Genomic analyses, although helpful in generating hypotheses about the natural 818 history of these species, will need to be paired with an in-depth understanding of natural 819 history, including integration of local knowledge, for managing these fish stocks. A 820 sustainable management approach is crucial to protect the inshore nursery habitats and 821 ensure recruitment of the species in Lake Tanganyika.

\section{Funding}

This work was supported by the Swiss National Science Foundation (grant CR23I2-166589 to OS), the University of Wyoming (start-up funding to CEW), the National Science Foundation (grant DEB-1556963 to CEW), the National Institute of General Medical Sciences of the National Institutes of Health (Institutional Development Award grant P20GM103432), and the American Genetics Association (Ecological, Evolutionary, and Conservation

\section{Genomics Research Award to JAR).}

\section{Acknowledgements}

832 Computing was accomplished with an allocation from the University of Wyoming's Advanced 833 Research Computing Center, on its Teton Intel x86_64 cluster 834 (https://doi.org/10.15786/M2FY47) and the Genetic Diversity Center (GDC) of ETH Zürich. 835 Special thanks go to Benedikt Ehrenfels for coordinating field campaigns, Mupape Mukuli for 836 facilitating logistics during fieldwork, and to the crew of the MV Maman Benita for fieldwork 837 assistance. We thank the whole team at the Tanzanian Fisheries Research Institute for their 838 support. A special thanks goes to Mary Kishe for her support during fieldwork permission 
839 processes and to the Tanzanian Commission for Science and Technology (COSTECH) for

840 the permits to do this research. Live fish samples were collected under the approved

841 University of Wyoming IACUC protocol \#20160602CW00241-01. We appreciate The Nature

842 Conservancy's Tuungane Project for providing funding and logistical support, with special

843 thanks going to Colin Apse and Peter Limbu. Thanks to members of the Wagner lab at the

844 University of Wyoming and the FishEc group at EAWAG for discussions about the analyses

845 and manuscript. We also thank the Associate Editor and four anonymous reviewers for the

846 valuable comments that greatly improved the quality of this manuscript.

\section{Data Availability}

850 We have deposited the primary data underlying these analyses as follows:

851 - Sampling locations, morphological data, filtered VCF files have been archived on Zenodo (http://doi.org/10.5281/zenodo.5216259)

853 - Fastq files for each individual have been archived in NCBI SRA XXXXX

854 - Scripts for data analysis are available on Github (https://github.com/jessicarick/lates855 popgen), and the version at publication will be archived on Zenodo 


\section{References}

Anderson, EC (2018) whoa: Where's my Heterozygotes? Observations on genotyping Accuracy. Version 0.0.2.999. Zenodo, doi: 10.5281/zenodo.2401764

Baird NA, Etter PD, Atwood TS, et al. (2008) Rapid SNP discovery and genetic mapping using sequenced RAD markers. PLoS One 3.

Bay LK, Crozier RH, Caley MJ (2006) The relationship between population genetic structure and pelagic larval duration in coral reef fishes on the Great Barrier Reef. Marine Biology 149, 1247-1256.

Berg PR, Star B, Pampoulie C, et al. (2017) Trans-oceanic genomic divergence of Atlantic cod ecotypes is associated with large inversions. Heredity 119, 418-428.

Bergamino N, Horion S, Stenuite S, et al. (2010) Spatio-temporal dynamics of phytoplankton and primary production in Lake Tanganyika using a MODIS based bio-optical time series. Remote Sensing of Environment 114, 772-780.

Bernatchez $L$ (2016) On the maintenance of genetic variation and adaptation to environmental change: considerations from population genomics in fishes. Journal of Fish Biology 89, 2519-2556.

Brannon EL, Powell MS, Quinn TP, Talbot A (2004) Population Structure of Columbia River Basin Chinook Salmon and Steelhead Trout. Reviews in Fis heries Science 12, 99232.

Bulengela G, Onyango P, Brehm J, et al. (2020) "Bring fishermen at the center": the value of local knowledge for understanding fisheries resources and climate-related changes in Lake Tanganyika. Environment, Development and Sustainability 22, 5621-5649.

Catchen J, Hohenlohe PA, Bassham S, Amores A, Cresko WA (2013) Stacks: an analysis tool set for population genomics. Mol Ecol 22, 3124-3140.

Chang CC, Chow CC, Tellier LCAM, et al. (2015) Second-generation PLINK: rising to the challenge of larger and richer datasets. Gigascience 4, s13742-015-0047-8.

Chapman DW (1976) Acoustic Estimates of Pelagic Ichthyomass in Lake Tanganyika with an Inexpensive Echo Sounder. Transactions of the American Fisheries Society 105, 581587.

Cirhuza DM, Plisnier PD (2016) Composition and seasonal variations in abundance of Copepod (Crustacea) populations from the northern part of Lake Tanganyika. Aquatic Ecosystem Health \& Management 19, 401-410.

Cohen AS, Gergurich EL, Kraemer BM, et al. (2016) Climate warming reduces fish production and benthic habitat in Lake Tanganyika, one of the most biodiverse freshwater ecosystems. Proc Natl Acad Sci U S A 113, 9563-9568.

Cohen AS, Lezzar K-E, Tiercelin J-J, Soreghan M (1997) New palaeogeographic and lakelevel reconstructions of Lake Tanganyika: implications for tectonic, climatic and biological evolution in a rift lake. Basin Research 9, 107-132.

Cohen AS, Soreghan MJ, Scholz CA (1993) Estimating the age of formation of lakes: an example from Lake Tanganyika, East African Rift system. Geology 21, 511-514.

Coulter GW (1970) Population changes within a group of fish species in Lake Tanganyika following their exploitation. Journal of Fish Biology 2, 329-353.

Coulter GW (1976) The biology of Lates species (Nile perch) in Lake Tanganyika, and the status of the pelagic fishery for Lates species and Luciolates stappersii (Blgr.). Journal of Fish Biology 9, 235-259.

Coulter GW (1991) Lake Tanganyika and its Life British Museum (Natural History) Cromwell Road, London SW7 5BD \& Oxford University Press, Walton Street, Oxford OX2 6DP.

Dahle G, Johansen T, Westgaard J-I, Aglen A, Glover KA (2018) Genetic management of mixed-stock fisheries "real-time": The case of the largest remaining cod fishery operating in the Atlantic in 2007-2017. Fisheries Research 205, 77-85.

Danecek P, Auton A, Abecasis G, et al. (2011) The variant call format and VCFtools. Bioinformatics 27, 2156-2158.

De Keyzer ELR, De Corte Z, Van Steenberge M, et al. (2019) First genomic study on Lake Tanganyika sprat Stolothrissa tanganicae: a lack of population structure calls for 
integrated management of this important fisheries target species. BMC Ecology and Evoluiton 19, 6.

De Keyzer ELR, Mulungula PM, Lufungula GA, et al. (2020) Local perceptions on the state of the pelagic fisheries and fisheries management in Uvira, Lake Tanganyika, DR Congo. Journal of Great Lakes Research 46, 1740-1753.

Dray S, Dufour A-B (2007) The ade4 Package: Implementing the Duality Diagram for Ecologists. 2007 22, 20.

Duftner N, Koblmüller S, Sturmbauer C (2005) Evolutionary relationships of the Limnochromini, a tribe of benthic deepwater cichlid fish endemic to Lake Tanganyika, East Africa. Journal of Molecular Evolution 60, 277-289.

Eccles DH (1992) Field guide to the freshwater fishes of Tanzania. In: species identification sheets for fishery purposes (ed. FAO), p. 145. FAO, Rome.

Ellegren H, Galtier N (2016) Determinants of genetic diversity. Nature Reviews Genetics 17, $422-433$.

Ellis CMA (1978) Biology of Luciolates-Stappersi in Lake Tanganyika (Burundi). Transactions of the American Fisheries Society 107, 557-566.

Genner MJ, Nichols P, Shaw PW, et al. (2010) Population structure on breeding grounds of Lake Malawi's 'twilight zone' cichlid fishes. Journal of Biogeography 37, 258-269.

Gompert Z, Lucas LK, Buerkle CA, et al. (2014) Admixture and the organization of genetic diversity in a butterfly species complex revealed through common and rare genetic variants. $\mathrm{Mol} \mathrm{Ecol} 23,4555-4573$.

Greenwood, PH (1981) Species-flocks and explosive evolution. Chance, change and challenge- the evolving biosphere, 61-74.

Gruber B, Unmack PJ, Berry OF, Georges A (2018) dartr: An r package to facilitate analysis of SNP data generated from reduced representation genome sequencing. Molecular Ecology Resources 18, 691-699.

Hutchinson WF (2008) The dangers of ignoring stock complexity in fishery management: the case of the North Sea cod. Biol Lett 4, 693-695.

Ivory SJ, McGlue MM, Peterman C, et al (2021) Climate, vegetation, and weathering across space and time in Lake Tanganyika (tropical eastern Africa). Quaternary Science Advances 3, 10023.

Janzen T, Etienne RS (2017) Inferring the role of habitat dynamics in driving diversification: evidence for a species pump in Lake Tanganyika cichlids.

Johnson MS, Black R (1984) Pattern beneath the chaos: The effect of recruitment on genetic patchiness in an intertidal limpet. Evolution 38, 1371-1383.

Junker J, Rick JA, Mclntyre PB, et al. (2020) Structural genomic variation leads to genetic differentiation in Lake Tanganyika's sardines. Mol Ecol 29, 3277-3298.

Kimirei IA, Mgaya YD, Chande Al (2008) Changes in species composition and abundance of commercially important pelagic fish species in Kigoma area, Lake Tanganyika, Tanzania. Aquatic Ecosystem Health and Management 11, 29-35.

Kimirei IA, Sweke EA (2018) Community-based conservation areas as a fisheries management tool in the East African Great Lakes: an example from Tanzania. In: $34^{\text {th }}$ SIL Congress, 19-24 August 2018. Nanjing, China. pp. 230 Book of abstracts.

Kirubakaran TG, Grove H, Kent MP, et al. (2016) Two adjacent inversions maintain genomic differentiation between migratory and stationary ecotypes of Atlantic cod. Mol Ecol 25, 2130-2143.

Kmentová N, Koblmüller S, Van Steenberge M, et al. (2020) Weak population structure and recent demographic expansion of the monogenean parasite Kapentagyrus spp. infecting clupeid fishes of Lake Tanganyika, East Africa. International Journal for Parasitology 50, 471-486.

Knaus BJ, Grünwald NJ (2017) VCFR: a package to manipulate and visualize variant call format data in R. Mol Ecol Resources 17, 44-53.

Koblmüller S, Duftner N, Katongo C, et al. (2005) Ancient divergence in bathypelagic Lake Tanganyika deepwater cichlids: Mitochondrial phylogeny of the tribe Bathybatini. Journal of Molecular Evolution 60, 297-314. 
966

967

968

969

970

971

972

973

974

975

976

977

978

979

980

981

982

983

984

985

986

987

988

989

990

991

992

993

994

995

996

997

998

999

1000

1001

1002

1003

1004

1005

1006

1007

1008

1009

1010

1011

1012

1013

1014

1015

1016

1017

1018

1019

1020

1021

Koblmüller S, Odhiambo EA, Sinyinza D, et al. (2015) Big fish, little divergence: phylogeography of Lake Tanganyika's giant cichlid, Boulengerochromis microlepis. Hydrobiologia 748, 29-38.

Koblmüller S, Zangl L, Börger C, et al. (2019) Only true pelagics mix: comparative phylogeography of deepwater bathybatine cichlids from Lake Tanganyika. Hydrobiologia 832, 93-103.

Koblmüller S, Schöggl CA, Lorber CJ, et al. (2021) African lates perches (Teleostei, Latidae, Lates): Paraphyly of Nile perch and recent colonization of Lake Tanganyika. Molecular Phylogenetics and Evolution 160, 107141.

Korneliussen TS, Albrechtsen A, Nielsen R (2014) ANGSD: Analysis of Next Generation Sequencing Data. BMC Bioinformatics 15, 356.

Korneliussen TS, Moltke I, Albrechtsen A, Nielsen R (2013) Calculation of Tajima's D and other neutrality test statistics from low depth next-generation sequencing data. BMC Bioinformatics 14, 289.

Kurki H, Mannini P, Vuorinen I, et al. (1999) Macrozooplankton communities in Lake Tanganyika indicate food chain differences between the northern part and the main basins. Hydrobiologia 407, 123-129.

Kuusipalo L (1999) Genetic differentiation of endemic nile perch Lates stappersi (Centropomidae, Pisces) populations in Lake Tanganyika suggested by RAPD markers. In: Lindqvist O.V., Mölsä H., Salonen K., Sarvala J. (eds) From Limnology to Fisheries: Lake Tanganyika and Other Large Lakes. Developments in Hydrobiology, vol 141. Springer, Dordrecht.

Langmead B, Salzberg SL (2012) Fast gapped-read alignment with Bowtie 2. Nat Methods 9, 357-359.

Lewis PO (2001) A Likelihood Approach to Estimating Phylogeny from Discrete Morphological Character Data. Systematic Biology 50, 913-925.

$\mathrm{Li} \mathrm{H}$, Durbin R (2009) Fast and accurate short read alignment with Burrows-Wheeler transform. Bioinformatics (Oxford, England) 25, 1754-1760.

Li H, Handsaker B, Wysoker A, et al. (2009) The Sequence Alignment/Map format and SAMtools. Bioinformatics 25, 2078-2079.

Loiselle S, Cózar A, Adgo E, et al. (2014) Decadal Trends and Common Dynamics of the Bio-Optical and Thermal Characteristics of the African Great Lakes. PLoS One 9, e93656.

Manier MK, Arnold SJ (2006) Ecological correlates of population genetic structure: a comparative approach using a vertebrate metacommunity. Proceedings of the Royal Society B: Biological Sciences 273, 3001-3009.

Mannini P (1998a) Ecology of the Pelagic Fish Resources of Lake Tanganyika, University of Hull.

Mannini P, Aro E, Katonda I, et al. (1996) Pelagic fish stocks of Lake Tanganyika: biology and exploitation. FAO/FINNIDA Research for the Management of the Fisheries of Lake Tanganyika. In: GCP/RAF/271/FIN-TD/53 (En), p. 60p.

Mannini P, Katonda I, Kissaka B, Verburg P (1999) Feeding ecology of Lates stappersii in Lake Tanganyika. Hydrobiologia 407, 131-139.

Martinez Barrio A, Lamichhaney S, Fan G, et al. (2016) The genetic basis for ecological adaptation of the Atlantic herring revealed by genome sequencing. Elife 5 .

McGlue MM, Ivory SJ, Stone JR, et al. (2020) Solar irradiance and ENSO affect food security in Lake Tanganyika, a major African inland fishery. Science Advances 6, eabb2191.

Meisner J, Liu S, Huang M, Albrechtsen A (2021) Large-scale inference of population structure in presence of missingness using PCA. Bioinformatics, btab027.

Mölsä H, Reynolds JE, Coenen EJ, Lindqvist OV (1999) Fisheries research towards resource management on Lake Tanganyika. Hydrobiologia 407, 1-24.

Mölsä H, Sarvala J, Badende S, et al. (2002) Ecosystem monitoring in the development of sustainable fisheries in Lake Tanganyika. Aquatic Ecosystem Health and Management 5, 267-281.

Mulimbwa N, Raeymaekers JAM, Sarvala J (2014) Seasonal changes in the pelagic catch of two clupeid zooplanktivores in relation to the abundance of copepod zooplankton in 
the northern end of Lake Tanganyika. Aquatic Ecosystem Health \& Management 17, 25-33.

Muller P, Li XP, Niyogi KK (2001) Non-photochemical quenching. A response to excess light energy. Plant Physiol 125, 1558-1566.

Munyandero J (2002) The lake Tanganyika clupeid and latid fishery system: Indicators and problems inherent in assessments and management. African Study Monographs 23, 117-145.

Mziray P, Kimirei IA, Staehr PA, et al. (2018) Seasonal patterns of thermal stratification and primary production in the northern parts of Lake Tanganyika. Journal of Great Lakes Research 44, 1209-1220.

O'Reilly CM, Alin SR, Piisnier PD, Cohen AS, McKee BA (2003) Climate change decreases aquatic ecosystem productivity of Lake Tanganyika, Africa. Nature 424, 766-768.

Ogutu-Ohwayo R, Natugonza V, Musinguzi L, et al. (2016) Implications of climate variability and change for African lake ecosystems, fisheries productivity, and livelihoods. Journal of Great Lakes Research 42, 498-510.

Olsen JB, Crane PA, Flannery BG, et al. (2011) Comparative landscape genetic analysis of three Pacific salmon species from subarctic North America. Conservation Genetics 12, 223-241.

Parchman TL, Gompert Z, Mudge J, et al. (2012) Genome-wide association genetics of an adaptive trait in lodgepole pine. Mol Ecol 21, 2991-3005.

Peterman WE, Anderson TL, Ousterhout BH, et al. (2015) Differential dispersal shapes population structure and patterns of genetic differentiation in two sympatric pond breeding salamanders. Conservation Genetics 16, 59-69.

Petit P, Shipton T (2012) IUU Fishing on Lake Tanganyika. Programme for the Implementation of a Regional Fisheries Strategy for the Eastern and Southern Africa and Indian Ocean Region.

Pettersson ME, Rochus CM, Han F, et al. (2019) A chromosome-level assembly of the Atlantic herring genome-detection of a supergene and other signals of selection. Genome Research 29, 1919-1928.

Plisnier PD, Chitamwebwa D, Mwape L, et al. (1999) Limnological annual cycle inferred from physical-chemical fluctuations at three stations of Lake Tanganyika. Hydrobiologia 407, 45-58.

Plisnier PD, Mgana H, Kimirei I, et al. (2009) Limnological variability and pelagic fish abundance (Stolothrissa tanganicae and Lates stappersii) in Lake Tanganyika. Hydrobiologia 625,117-134.

Pritchard JK, Stephens M, Donnelly P (2000) Inference of population structure using multilocus genotype data. Genetics 155: 945-959.

Puechmaille SJ (2016) The program STRUCTURE does not reliably recover the correct population structure when sampling is uneven: subsampling and new estimators alleviate the problem. Molecular Ecology Resources 16, 608-627.

Purcell S, Neale B, Todd-Brown K, et al. (2007) PLINK: a toolset for whole-genome association and population-based linkage analysis. American Journal of Human Genetics, 81: 559-575.

R Core Team (2021) R: A Language and Environment for Statistical Computing. R Foundation for Statistical Computing, Vienna, Austria. https://www.R-project.org/.

Reich D, Thangaraj K, Patterson N, Price AL, Singh L (2009) Reconstructing Indian population history. Nature 461, 489-494.

Reiss H, Hoarau G, Dickey-Collas M, Wolff WJ (2009) Genetic population structure of marine fish: mismatch between biological and fisheries management units. Fish and Fisheries 10, 361-395.

Riginos C, Buckley YM, Blomberg SP, et al. (2014) Dispersal Capacity Predicts Both Population Genetic Structure and Species Richness in Reef Fishes. The American Naturalist 184, 52-64.

Riginos C, Doublas KE, Jin Y, et al. (2011) Effects of geography and life history traits on genetic differentiation in benthic marine fishes. Ecography 34, 566-575. 
Sarvala J, Tarvainen M, Salonen K, Mölsä H (2002) Pelagic food web as the basis of fisheries in Lake Tanganyika: A bioenergetic modeling analysis. Aquatic Ecosystem Health and Management 5, 283-292.

Sayers M, Bosse K, Fahnenstiel G, Shuchman R (2020) Carbon fixation trends in eleven of the world's largest lakes: 2003-2018. Water 12, 3500.

Schindler DE, Armstrong JB, Reed TE (2015) The portfolio concept in ecology and evolution. Frontiers in Ecology and the Environment 13, 257-263.

Schindler DE, Hilborn R, Chasco B, et al. (2010) Population diversity and the portfolio effect in an exploited species. Nature 465, 609-612.

Sedano RE, Burns KJ, Riddle B (2010) Are the Northern Andes a species pump for Neotropical birds? Phylogenetics and biogeography of a clade of Neotropical tanagers (Aves: Thraupini). Journal of Biogeography 37, 325-343.

Selkoe KA, Toonen RJ (2011) Marine connectivity: a new look at pelagic larval duration and genetic metrics of dispersal. Marine Ecology Progress Series 436, 291-305.

Shanks (2009) Pelagic larval duration and dispersal distance revisited. The Biological Bulletin 216, 373-385.

Shastry, V, Adams PE, Lindtke D, et al. (2021) Model-based genotype ancestry estimation for potential hybrids with mixed-ploidy. Mol Ecol Resources 21: 1434-1451.

Stamatakis A (2014) RAxML version 8: a tool for phylogenetic analysis and post-analysis of large phylogenies. Bioinformatics 30, 1312-1313.

Stearns SC (1992) The evolution of life histories Oxford Univ. Press, New York.

Stenuite S, Pirlot S, Hardy MA, et al. (2007) Phytoplankton production and growth rate in Lake Tanganyika: evidence of a decline in primary productivity in recent decades. Freshwater Biology 52, 2226-2239.

Sterner T (2007) Unobserved diversity, depletion and irreversibility The importance of subpopulations for management of cod stocks. Ecological Economics 61, 566-574.

Sturmbauer C, Börger C, Van Steenberge M, Koblmüller S (2016) A separate lowstand lake at the northern edge of Lake Tanganyika? Evidence from phylogeographic patterns in the cichlid genus Tropheus. Hydrobiologia 791, 51-68.

Sunday JM, Popovic I, Palen WJ, Foreman MG, Hart MW (2014) Ocean circulation model predicts high genetic structure observed in a long-lived pelagic developer. $\mathrm{Mol} \mathrm{Ecol}$ 23, 5036-5047.

Supple MA, Shapiro B (2018) Conservation of biodiversity in the genomics era. Genome Biology 19, 131.

Tange O (2011) GNU Parallel - The Command-Line Power Tool. ;login: The USENIX Magazine, 36:42-47.

[dataset] Temasek Life Sciences Laboratory (2016). Lates calcarifer isolate:ASB-BC8 Genome sequencing and assembly. National Center for Biotechnology Information Sequence Read Archive, Assembly GCA_001640805.1.

Therkildsen NO, Hemmer-Hansen J, Als TD, et al. (2013) Microevolution in time and space: SNP analysis of historical DNA reveals dynamic signatures of selection in Atlantic cod. Mol Ecol 22, 2424-2440.

Van der Knaap M (2013) Comparative analysis of fisheries restoration and public participation in Lake Victoria and Lake Tanganyika. Aquatic Ecosystem Health and Management 16, 279-287.

Van der Knaap M, Katonda KI, De Graaf GJ (2014) Lake Tanganyika fisheries frame survey analysis: Assessment of the options for management of the fisheries of Lake Tanganyika. Aquatic Ecosystem Health and Management 17, 4-13.

van Zwieten PAM, Roest FC, Machiels MAM, Van Densen WLT (2002) Effects of interannual variability, seasonality and persistence on the perception of long-term trends in catch rates of the industrial pelagic purse-seine fishery of northern Lake Tanganyika (Burundi). Fisheries Research 54, 329-348.

van Zwieten PAM, Kolding J, Plank MJ, et al. (2016) The Nile perch invasion in Lake Victoria: cause or consequence of the haplochromine decline? Canadian Journal of Fisheries and Aquatic Sciences 73, 622-643. 
Verburg P, Antenucci JP, Hecky RE (2011) Differential cooling drives large-scale convective circulation in Lake Tanganyika. Limnology and Oceanography 56, 910-926.

Verburg P, Hecky RE (2009) The physics of the warming of Lake Tanganyika by climate change. Limnology and Oceanography 54, 2418-2430.

Verburg P, Hecky RE, Kling H (2003) Ecological consequences of a century of warming in Lake Tanganyika. Science 301, 505-507.

Vij S, Kuhl H, Kuznetsova IS, et al. (2016) Chromosomal-Level Assembly of the Asian Seabass Genome Using Long Sequence Reads and Multi-layered Scaffolding. PLOS Genetics 12, e1005954-e1005954.

Wang, J (2017) The computer program STRUCTURE for assigning individuals to populations: easy to use but easier to misuse. Molecular Ecology Resources 17, 981990.

Wenburg JK, Bentzen P, Foote CJ (1998) Microsatellite analysis of genetic population structure in an endangered salmonid: the coastal cutthroat trout (Oncorhynchus clarki clarki). Molecular Ecology 7, 733-749.

White JW, Botsford LW, Hastings A, Largier JL (2010) Population persistence in marine reserve networks: incorporating spatial heterogeneities in larval dispersal. Marine Ecology Progress Series 398, 49-67.

Young EF, Belchier M, Hauser L, et al. (2015) Oceanography and life history predict contrasting genetic population structure in two Antarctic fish species. Evolutionary Applications 8, 486-509. 